\title{
A Rare Malignant Transformation of Benign Lacrimal Tumor
}

\author{
Rajan Kumar Labh, ${ }^{1}$ Gulshan Bahadur Shrestha ${ }^{2}$ \\ 'Biratnagar Eye Hospital, Biratnagar, Morang, ${ }^{2}$ BP Koirala Lion's Center for Ophthalmic Studies, Kathmandu, Nepal.
}

\begin{abstract}
Lacrimal gland tumors are common in middle age group individuals. Proptosis is one of their common presentations. Sometimes the tumors may undergo malignant changes which if neglected can be life threatening. Here we present a case of 49 years male with gradual painless protrusion and diminution of vision in his right eye for eight years and more since last 2 months, with restriction of ocular motility in right lateral gaze. Expecting a benign mass, superolateral orbitotomy with excisional biopsy was done which astonishingly revealed carcinoma ex pleomorphic adenoma with components of adenocarcinoma and squamous cell carcinoma without any lymphovascular invasion. Proptosis and vision of the patient in right eye improved post excisional biopsy without any residual mass in respective follow ups.
\end{abstract}

Keywords: adenocarcinoma; lacrimal gland; malignant transformation; proptosis.

\section{INTRODUCTION}

The lacrimal glands, a tubuloacinar glands, one for each eye, secretes the aqueous layer of the tear film. ${ }^{1}$ Lacrimal glandlesions includes infiltrative processes, structural disorders, and epithelial neoplasms. ${ }^{2-5}$ Epithelial tumors represent $20 \%$ of all lacrimal gland lesions. Among the epithelial lesions, $55 \%$ are benign and $45 \%$ are malignant. ${ }^{6}$ Pleomorphic adenomas are most common benign lesion whereas carcinoma ex pleomorphic adenoma is the second most common malignant lesion with variable prognosis. ${ }^{6}$ This case report basically shows the malignant transformation of their benign counterpart in short span of time that sometimes can go overlooked. Hence, there is need for detail evaluation in any cause of proptosis, especially with recent change in its presentations. Since no similar cases have been reported from Nepal, this case report may help others in understanding and diagnosing such cases.

\section{CASE REPORT}

A 49 years male presented with gradual painless protrusion and diminution of vision in right eye for 8 years, more since last 2 months (Figure 1).

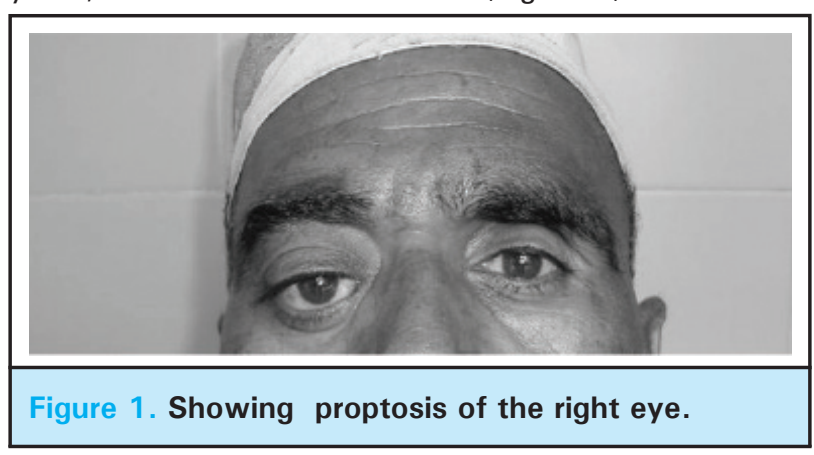

There was no associated history of trauma, redness, discharge, double vision, flashes and floaters. Vision in right and left eye was 6/36 (pinhole-6/12) and 6/6 respectively.Examinations revealed nonaxial proptosis and mild aponeurotic ptosis of right eye with restriction of ocular movement in the right lateral gaze. There

Correspondence: Dr. Rajan Kumar Labh, Biratnagar Eye Hospital, Rani, Biratnagar, Nepal. Email:rajan.labh@yahoo.com, Phone: 9841390810. 
was $1 \times 0.5 \mathrm{~cm}$ nontender, firm, immobile, irreducible, noncompressible mass in the superotemporal quadrant of the right orbit free from underlying skin anteriorly while posterior extent was unappreciable. Ptosis was mild with good levatorpalpebrae function, probably because of mechanical and aponeuroticcomponents. The sclera and cornea was normal, and the pupils were bilaterally symmetrical and reacting to light. Fundoscopy was normal. Lymphnodes, cranial nerves and systemic examination were normal. The left eye was normal and there was no intracranial focal lesion. CT scan imaging of the head and orbit revealed heterogenously enhancing soft tissue extraconal mass in the right superolateral quadrant of the orbit most likely mass of lacrimal gland origin (Figure 2).

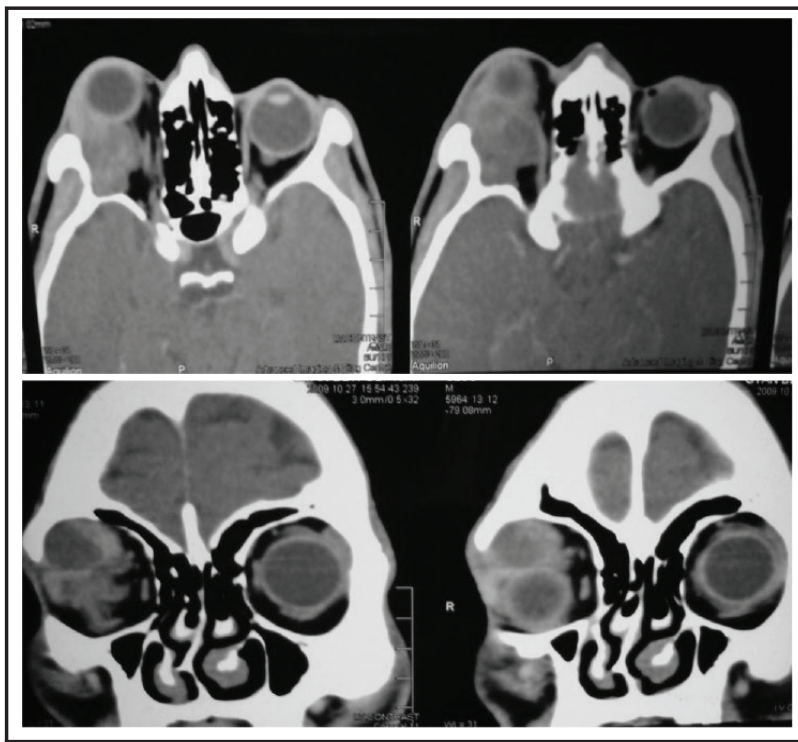

Figure 2. CT axial and coronal section of the brain and orbit showing heterogenously enhancing attenuated extraconal mass measuring $30.5 \mathrm{~mm} \times 23.5 \mathrm{~mm}$ in size in superolateral quadrant of the right orbit with small faint calcification within it. Right lacrimal gland is not seen separated from this mass.

Chest X-ray and abdominal ultrasonography was normal. Expecting a benign mass of lacrimal gland, which is quite common finding, right eye superolateral orbitotomy with excisional biopsy was planned and done. Unexpectedly histopathological examination of the mass revealed intracapsular carcinoma in pleomorphic adenoma/carcinoma ex pleomorphic adenoma with component of adenocarcinoma and squamous cell carcinoma without any lymphovascular invasion (Figure 3-4). Though the histology report was unfavourable, patient improved post excisional biopsy and in respective follow ups without any residual mass.

\section{DISCUSSION}

Orbit is a bony structure covered on all sides by bony walls except anteriorly. The diseases in the orbit can arise primarily from the orbit itself or secondarily from surrounding regions like paranasal sinuses, eyelids, periorbital area and intracranial compartment.

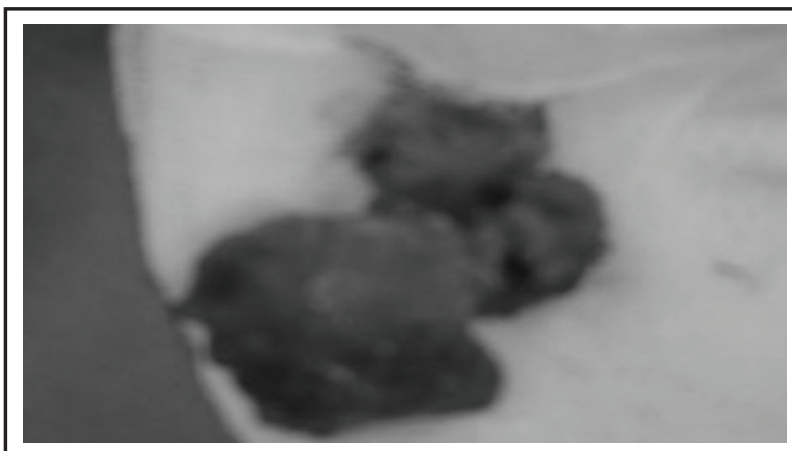

Figure 3. Showing gross well circumscribed, lobulated and solid mass measuring $4 \times 3 \mathrm{~cm}$ post excision .

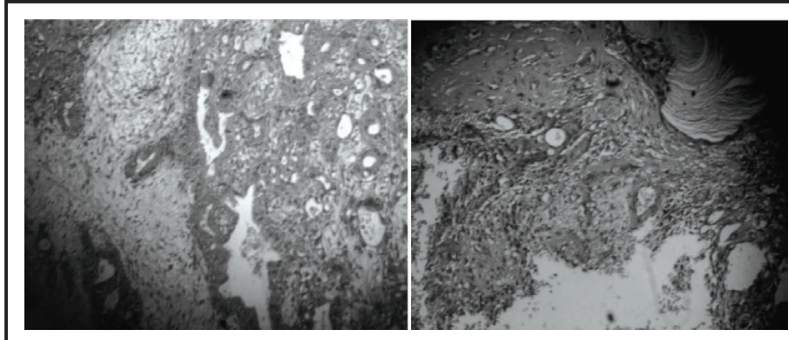

Figure 4. Microscopic section shows tumor composed of epithelial and myoepithelial cells forming tubules and sheets with fibromyxoid stroma. The cytoplasm is eosinophilic with large,hyperchromatic and pleomorphic nuclei. Some of the tumor cells also show irregular nuclear membrane. Mitotic figures are also seen. Focal area of squamous differentiation with keratinization is also present.

Thus, ophthalmic symptoms are common presenting findings with most orbital diseases. The lacrimal gland is considered to be a minor salivary gland that shares histologic features with the major salivary glands. Hence, in many respects, lacrimal gland tumors resemble those that arise in the major salivary glands. ${ }^{7}$

Only few studies has been published regarding clinicpathological characteristic of carcinoma ex pleomorphic adenoma. It comprises only $0.3 \%$ of all lacrimal gland tumors and is the second most common malignant tumor with incidence of around $18 \% .{ }^{8}$ It is more commoner in $6^{\text {th }}$ to $7^{\text {th }}$ decade of life. ${ }^{8}$ The origin is usually from aged pleomorphic adenoma or recurrent pleomorphic adenoma after excision. Painless proptosis is the most common presentation. However, some 
unusual presentations like abrupt orbital inflammation or a painful subcutaneous nodule have been reported. ${ }^{8}$ The diagnosis is based on histopathological examination and Computed Tomography, Magnetic Resonance Imaging (MRI) or orbital echography. ${ }^{6,7}$ Histopathologically, the tumor can be classified into noninvasive (intracapsular), minimally invasive $(<1.5 \mathrm{~mm}$ from the capsule) and invasive $(>1.5 \mathrm{~mm}$ from the capsule). Noninvasive and minimally invasive carcinomas have excellent prognosis with complete surgical excision and without adjunctive radiotherapy. On the contrary, invasive carcinoma ex pleomorphic adenoma are aggressive and have poor prognosis. ${ }^{8}$ The extent of invasion,proportion of carcinoma, lymph node stage, histological grade affects the overall survival. ${ }^{6-8}$ Favourable prognosis was anticipated in our case because there was no invasion and proportion of cancer within the lesion was small. To conclude proptosis is one of the common causes for ocular morbidity. Sometimes, there may be malignant component in proptosed eye especially in adults with recent change in its presentation. Hence, detail history and workup can be prognostically very important and sometimes life saving for the patient.

\section{REFERENCES}

1. Keith LM, Arthur FD, Anne MRA. Clinically Oriented Anatomy, 6th ed. USA: Lippincott Williams \& Wilkins; 2012.

2. Font RL, Gamel JW,Jakobiec F.A Epithelial tumors of the lacrimal gland: Ocular and adnexal tumors, Birmingham: Aesculapius;1978:787.

3. Goder GJ. Tumours of the lacrimal gland: Orbit. 1982;1:91-6.

4. Zimmerman LA, Sanders TE, Ackerman LV. Epithelial tumors of the lacrimal gland: prognostic and therapeutic significance of histologic types. IntOphthalmolClin. 1962; 2:337-67.

5. Lemke AJ, Hosten N, Neumann K, et al .Space occupying lesions of the lacrimal gland in CT and MRI exemplified by four cases. AktuelleRadiol. 1995;5:363-6.
6. Shields JA, Shields CL, Epstein JA, Scartozzi R, Eagle RC Jr. Review: primary epithelial malignancies of the lacrimal gland: the 2003 Ramon L. OphthalPlastReconstr Surg. 2004 Jan;20(1):10-21.

7. Bernardini FP, Devoto MH, Croxatto JO. Epithelial tumors of the lacrimal gland: an update. CurrOpinOphthalmol. 2008 Sep;19(5):409-13.

8. Sakuma T, Ohashi H, Yamamoto K, Kawano K. Carcinoma ex pleomorphic adenoma of the lacrimal gland: a case report. Jpn J Ophthalmol. 2008;52(1):67-8. 